\title{
Exosomal miR-122-5p is Related to the Degree of Myelosuppression Caused by Chemotherapy in Patients with Colorectal Cancer
}

Jinbao Chen, ${ }^{1, *}$

Wentao Wu, ${ }^{1, *}$ Xue He, Linlin Jia,' Jiahua Yang,' Xianke Si, 'Kun Yu,' Sen Li, ' Yanyan Qiu, ${ }^{\prime} \mathrm{Ke} \mathrm{Xu}{ }^{2}$ Peihao Yin, ${ }^{1,3}$ Yijun Cao,' Qiong $\mathrm{Li}^{4}{ }^{4}$ Wei $\mathrm{Li}^{1}$

'Department of General Surgery, Putuo Hospital, Shanghai University of

Traditional Chinese Medicine, Shanghai, People's Republic of China; ${ }^{2}$ Institute of Translational Medicine, Shanghai University, Shanghai, People's Republic of China; ${ }^{3}$ Interventional Cancer Institute of Chinese Integrative Medicine, Shanghai University of Traditional Chinese Medicine, Shanghai, People's Republic of China; ${ }^{4}$ Department of General Surgery, Institute of Fudan-Minhang Academic Health System, Minhang Hospital, Fudan University, Shanghai, People's Republic of China

*These authors contributed equally to this work
Purpose: As rapidly dividing cells are usually the target of anticancer chemotherapy, it is inevitable that rapidly dividing normal cells become damaged, with myelosuppressive effects being a serious side effect of this therapy. Many recent studies have found that exosomal microRNAs (miRNAs) are related to the occurrence of some diseases.

Patients and Methods: Small RNA sequencing was used to investigate differential exosomal miRNAs with the same expression trend between groups after chemotherapy: MildA (before chemotherapy in patients with mild myelosuppression) and MildB (after chemotherapy in patients with mild myelosuppression); SevereA (before chemotherapy in patients with severe myelosuppression) and SevereB (after chemotherapy in patients with severe myelosuppression). A Venn diagram was generated to screen exosomal miRNAs related to chemotherapy. Small RNA sequencing was also used to investigate differentially expressed exosomal miRNAs among these groups, and exosomal miRNAs related to myelosuppression after chemotherapy was explored using a Venn diagram. RT-qPCR was applied to further verify the sequencing results. We performed target gene prediction and functional analysis for candidate exosomal miRNAs.

Results: Compared with that in the MildA or SevereA group, an increase in exosomal miR$122-5 p$ was found in the MildB or SevereB group, and the expression level was lower in the SevereB group than in the MildB group. However, we found no notable difference in its expression level between the MildA and SevereA groups. Similar results were not obtained for the remaining miRNAs. RT-qPCR confirmed the screening results. Further analyses indicated that exosomal miR-122-5p targets CDK4 to inhibit the cell cycle.

Conclusion: The expression level of exosomal miR-122-5p in the serum of patients with colorectal cancer correlates with the severity of myelosuppression caused by chemotherapy, and miR-122-5p targets CDK4 to inhibit cell cycle progression.

Keywords: colorectal cancer, chemotherapy, myelosuppression, miR-122-5p

\section{Introduction}

Colorectal cancer ranks as the third most common cancer in the world and has high morbidity and mortality. ${ }^{1,2}$ At present, the 5-year relative survival of patients is $65 \%{ }^{3}$ Chemotherapy is generally used to treat malignant tumors. However, normal tissue can incur damage to various degrees, with the possible development of a series of adverse reactions, such as nephrotoxicity, gastrointestinal diseases, and myelosuppression. Although nephrotoxicity and chemoresistance can be greatly reduced with advanced new high-efficiency and low-toxicity chemotherapeutics, including gastrointestinal diseases, myelosuppression has not. ${ }^{4,5}$ Myelosuppression involves a decrease in the activity 
of blood cell precursors in the bone marrow. As chemotherapy drugs target rapidly proliferating cells, hematopoietic stem cells can be severely affected, resulting in a decrease in red blood cells (RBCs), white blood cells (WBCs) and platelets in the peripheral blood. As people with cancer are more susceptible to infection when myelosuppression occurs, protecting patients from chemotherapy-induced myelosuppression when treating colorectal cancer remains a huge issue.

Exosomes, membrane-bound vesicles with a diameter of 40-100 nm, are found in almost all biological fluids. ${ }^{6-8}$ As for proteins, various nucleic acids, including miRNAs, we figured them in the exosomal lumen. ${ }^{9}$ Noninvasive biomarkers and exosomal miRNAs have the potential to reveal disease states. For instance, a set of exosomal miRNAs can function as diagnostic biomarkers for colorectal cancer, including let-7a, miR-1229, and miR-1246, among others. ${ }^{10}$ Another set, miR-1290 and miR-375, functions as prognostic markers for castration-resistant prostate cancer. ${ }^{11}$ As a type of noncoding RNA, microRNAs regulate expression of target genes posttranscriptionally, functioning in cell proliferation. $^{12,13}$ One study showed that targeted delivery of miR-26a protected the host against myelosuppression caused by chemotherapy. ${ }^{14}$ However, exosomal miRNAs associated with myelosuppression after chemotherapy in colorectal cancer have not yet been described.

By using performing small RNA sequencing, RTqPCR, target prediction and functional analysis, we in this study determined that exosomal miR-122-5p is related to myelosuppression after chemotherapy.

\section{Materials and Methods}

\section{Recruitment of Patients and Collection of Blood Samples}

The participants comprised patients who accepted over 8 cycles of the XELOX chemotherapy regimen after being diagnosed via imagology and pathology with malignant tumors from 2017 to 2020 . The inclusion criteria were as follows: confirmation of malignant tumor by pathology or radiography; acceptance of 8 consecutive cycles of chemotherapy; Karnofsky performance status (KPS) $\geq 70$; age between 18 and 75 years; and no serious heart, liver, kidney, chemotherapy contraindications or other factors affecting drug evaluation. The exclusion criteria were as follows: recurrence or metastasis in the abdominal cavity and liver; obvious heart (a history of fearful heart issues including coronary heart disease, myocardial infarction), liver (obviously abnormal ALT, AST), or kidney (CKD stage $>2$ ) disease, mental illness, pregnancy or breast-feeding; and allergic physique or allergy to multiple drugs.

Peripheral blood was collected from the participants before and after chemotherapy, and serum was obtained through centrifugation at $3000 \mathrm{rpm}$ for $10 \mathrm{~min}$. All samples were kept at $-80{ }^{\circ} \mathrm{C}$ for subsequent experiments. Patients were divided into mild myelosuppression and severe myelosuppression groups according to changes in leukocytes, neutrophils, platelets, and hemoglobin in the blood after chemotherapy, whereby mild myelosuppression was defined as $2.0 \times 10^{9} / \mathrm{L} \leq$ leukocyte count $\leq 4.0 \times 10^{9} / \mathrm{L}, 1 \times 10^{9} / \mathrm{L} \leq$ granulocyte count $\leq 2 \times 10^{9} / \mathrm{L}, 80$ $\mathrm{g} / \mathrm{L} \leq$ hemoglobin $\leq 120 \mathrm{~g} / \mathrm{L}$ or $50 \times 10^{9} / \mathrm{L} \leq$ platelet count $\leq 100 \times 10^{9} / \mathrm{L}$ and severe myelosuppression as leukocyte count $<2.0 \times 10^{9} / \mathrm{L}$, granulocyte count $<1 \times 10^{9} / \mathrm{L}$, hemoglobin $<80 \mathrm{~g} / \mathrm{L}$ or platelet count $<50 \times 10^{9} / \mathrm{L}$.

The present study was approved by the Ethics Committee of Hospital of Shanghai University of TCM. Patients who participated in this research signed informed consent, and complete clinical data were available. This study was conducted in accordance with the Declaration of Helsinki.

\section{Exosome Isolation, RNA Extraction and Library Construction}

Exosome isolation and RNA extraction were carried out using an exoRNeasy Serum/Plasma Maxi Kit (QIAGEN GmbH, Hilden, Germany) in accordance with the manufacturer's instructions. RNA quantitation was achieved using a Nanodrop 2000 (Thermo Fisher Scientific Inc., USA). The length and integrity of the RNA were assessed with an Agilent 2100 Bioanalyzer (Agilent Technology, USA). After RNA quality assessment, a small RNA library was produced using total RNA from the samples with TruSeq Small RNA Sample Prep Kit (Cat. No. RS-200-0012, Illumina, USA) following the manufacturer's recommendations. Briefly, adapters were ligated to each end of the RNA and reverse transcribed to cDNA, after which PCR amplification was performed. PCR products ranging from 140 to $160 \mathrm{bp}$ were isolated as small RNA libraries. The libraries were ultimately sequenced using the Illumina HiSeq X Ten platform, and $150 \mathrm{bp}$ paired-end reads were generated. The small RNA sequencing and analysis were performed by OE Biotech Co., Ltd. (Shanghai, China).

\section{Bioinformatic Analysis of Small RNA Sequencing Results}

Reads were transformed to sequence data (raw data/reads) via base calling, and low-quality reads were filtered for subsequent analysis. For primary analysis, noncoding RNAs annotated 
such as rRNAs, tRNAs, and small nuclear RNAs (snRNAs) were filtered out. Identification of known miRNAs was performed through alignment with the miRBase v22 database (http://www.mirbase.org/), and known miRNA expression patterns in different samples were analyzed. Differentially expressed miRNAs were filtered with a threshold $\mathrm{p}$ value $<0.05$, and their targets were predicted with miRanda software. The $p$ value was assessed using the DEG algorithm in the R package. GO enrichment and KEGG pathway enrichment analyses of the miRNA target genes were carried out in $\mathrm{R}$ based on hypergeometric distribution. All sequencing analyses were performed via OE Biotech Co., Ltd. (Shanghai, China).

\section{RT-qPCR Validation of Exosomal miRNAs}

Expression of exosomal miRNAs (miR-10b-3p, miR-122-5p, miR-222-3p, miR-370-3p, miR-320b, miR-605-3p) was evaluated with a miScript PCR System (QIAGEN, Hilden, Germany). Reverse transcription reactions were carried out using a miScript II RT Kit (QIAGEN, Hilden, Germany), and miScript HiSpec Buffer for cDNA synthesis was used for the detection of mature miRNA. Quantitative real-time PCR was performed with a miScript SYBR Green PCR Kit (QIAGEN, Hilden, Germany). Mature miRNAs were identified via miScript Primer Assay. miR-39 was used as an external control. ${ }^{15}$ The average $\mathrm{Ct}$ value for each RNA was obtained from triplicate reactions. Expression levels of miRNAs were calculated using $2^{\wedge}(-\Delta \mathrm{Ct})$, and fold changes of miRNAs were obtained by comparison of expression between case and control groups.

\section{Statistical Analysis}

SPSS software (SPSS for Windows, Version 13.0; SPSS Inc., Chicago, IL) was employed for statistical analysis. Data are expressed as the mean \pm standard deviation (SD). The Kolmogorov-Smirnov test indicated that the data were normally distributed. The $t$-test was applied for analyzing comparisons between two groups. The $p$ value was attained using a two-tailed test, and $\mathrm{p}<0.05$ indicated a significant difference.

\section{Results \\ Screening Exosomal miRNAs}

We screened for exosomal miRNAs that are significantly associated with myelosuppression and that may be act as therapeutic targets. Before and after chemotherapy, we collected serum samples from 3 patients with mild myelosuppression and 3 with severe myelosuppression and divided them into MildA, MildB, SevereA, and SevereB groups. A total of 12 specimens were screened and analyzed by small RNA sequencing. The whole screening process consisted mainly of three steps (Figure 1). 1) We detected differential exosomal miRNAs before and after

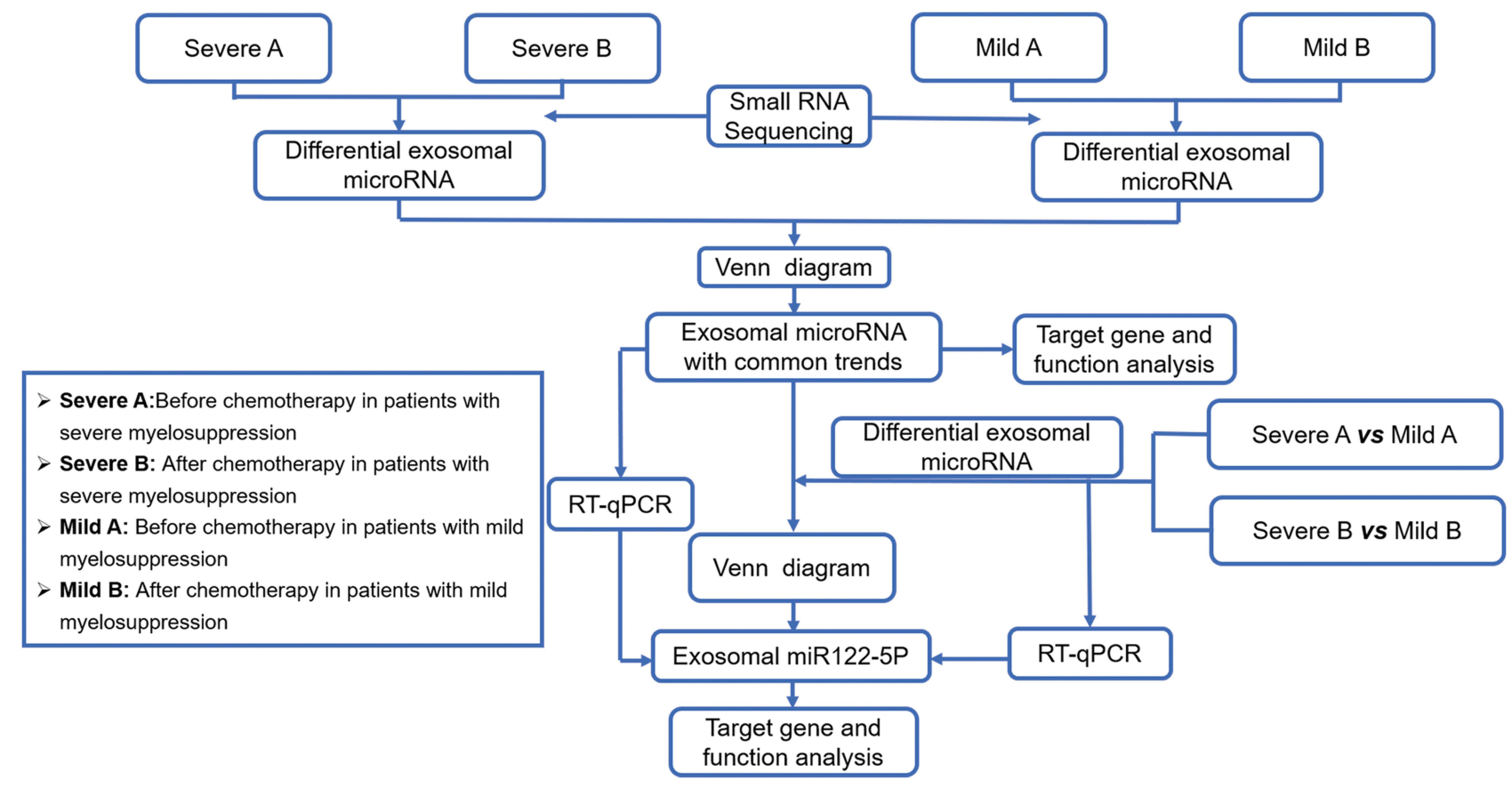

Figure I Schematic diagram of the research process. 
chemotherapy and screened out miRNAs with significant differences as candidates. Expression of these exosomal miRNAs was affected by chemotherapy. 2) We compared the groups of patients before and after chemotherapy (with differences in myelosuppression) and built a Venn diagram to screen differentially expressed exosomal miR-122-5p after chemotherapy. 3) We performed RT-qPCR to verify expression of exosomal miR-122-5p. Finally, target gene prediction and functional analysis of miR-122-5p were performed.

\section{Screening and Functional Analysis of Chemotherapy-Related Exosomal miRNAs}

To understand differences in expression of exosomal miRNAs before and after chemotherapy, we evaluated 6 patients and screened differentially expressed exosomal miRNAs. We detected 37 differentially expressed exosomal miRNAs in patients with severe myelosuppression (before chemotherapy: SevereA; after chemotherapy: SevereB) and 39 in patients with mild myelosuppression (before chemotherapy: MildA; after chemotherapy: Mild B). The expression levels are presented in a heatmap in Figure 2A and B. A Venn diagram was generated to compare differentially expressed exosomal miRNAs between the two mild and severe myelosuppression groups. The results revealed 6 exosomal miRNAs with the same expression trend that differed significantly after chemotherapy (three downregulated, miR-320b, miR-370$3 \mathrm{p}$, and $\mathrm{miR}-605-3 \mathrm{p}$, and three upregulated, miR-10b-3p, miR-122-5p, and miR-222-3p) (Figure 2C). The heatmap also shows 6 exosomal miRNAs with the same trend and differential expression among the groups (Figure 2D). GO and KEGG enrichment analyses of the 6 exosomal miRNA target genes indicated their functions, such as the cell cycle, cell motility and immune response (Figure $2 \mathrm{E}$ and F). These results by small RNA sequencing indicate that these 6 exosomal miRNAs are related to chemotherapy.

\section{Screening and Functional Analysis of Myelosuppression-Related Exosomal miR-I 22-5p}

To further understand exosomal miRNAs related to myelosuppression, we analyzed differentially expressed exosomal miRNAs between the SevereA and MildA groups and between the SevereB and MildB groups, with 52 differentially expressed miRNAs between the former groups and 69 between the latter (Figure 3A and B).
More interestingly, a Venn diagram showed exosomal miR-122-5p to be differentially expressed after chemotherapy, which was reflected mainly in nonsignificant differences in its expression between the MildA and SevereA groups and its strongly lower expression in the SevereB group than in the MildB group (Figure 3C). However, none of the exosomal miRNAs mentioned above exhibited differential expression related to chemotherapy. Thus, exosomal miR-122-5p has a certain association with the degree of myelosuppression in patients with colorectal cancer.

\section{Validation of Chemotherapy-Related Exosomal miRNAs by RT-qPCR}

We collected corresponding patient serum samples and used RT-qPCR to verify the differential expression of exosomal miRNAs. The results showed that the level of exosomal miR-122-5p in the SevereB group was higher than that in the SevereA group; the level of miR-122-5p in the MildB group was higher than that in the MildA group (Figure 4A and C). This is consistent with the small RNA sequencing results. However, for the remaining miRNAs (miR-10b-3p, miR-222-3p, miR-320b, miR-370-3p, and miR-605-3p), there was no significant difference in their level between the SevereA and SevereB groups or between the MildA and MildB groups, which was inconsistent with the small RNA sequencing results (Figure 4A-D). The findings (through small RNA sequencing and RT-qPCR) indicate that exosomal miR-122-5p is associated with chemotherapy.

\section{Validation of Myelosuppression-Related Exosomal miR-122-5p by RT-qPCR}

RT-qPCR was employed to further verify the differential expression of serum exosomal miRNA, confirming the results of small RNA sequencing (ie, no significant difference in level of exosomal miR-122-5p between SevereA and MildA but a higher level in MildB than in SevereB). Regardless, expression of other differentially expressed exosomal miRNAs did not differ significantly between the SevereB and MildB groups (Figure 5A-D). Based on verification using patient sera, we speculate that patients with high expression of exosomal miR-122-5p have milder myelosuppression after chemotherapy but that patients with low-expression experience more severe myelosuppression after chemotherapy. 
A

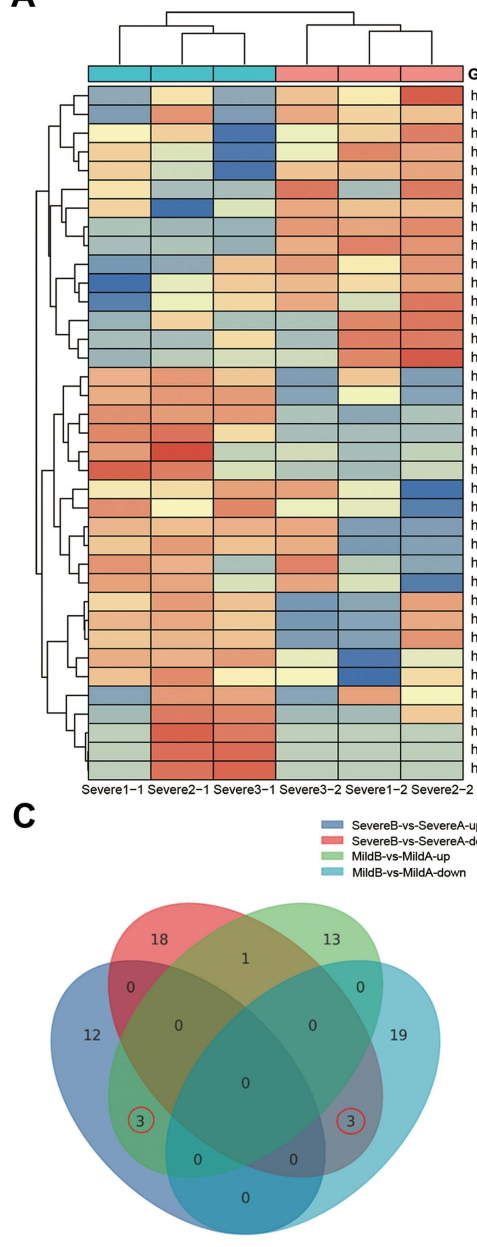

$\mathbf{E}$

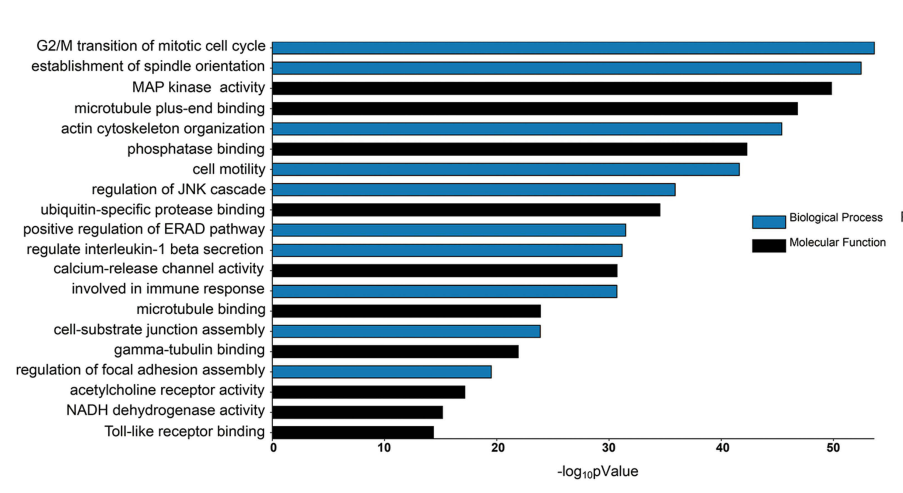

$\mathbf{F}$
B

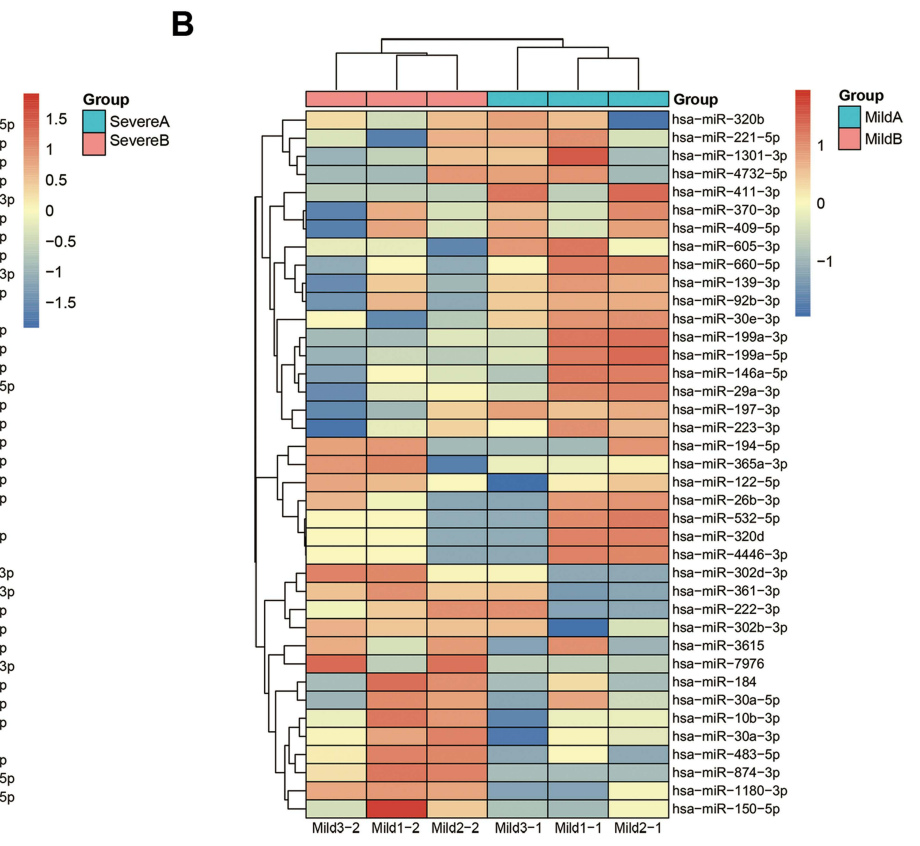

D
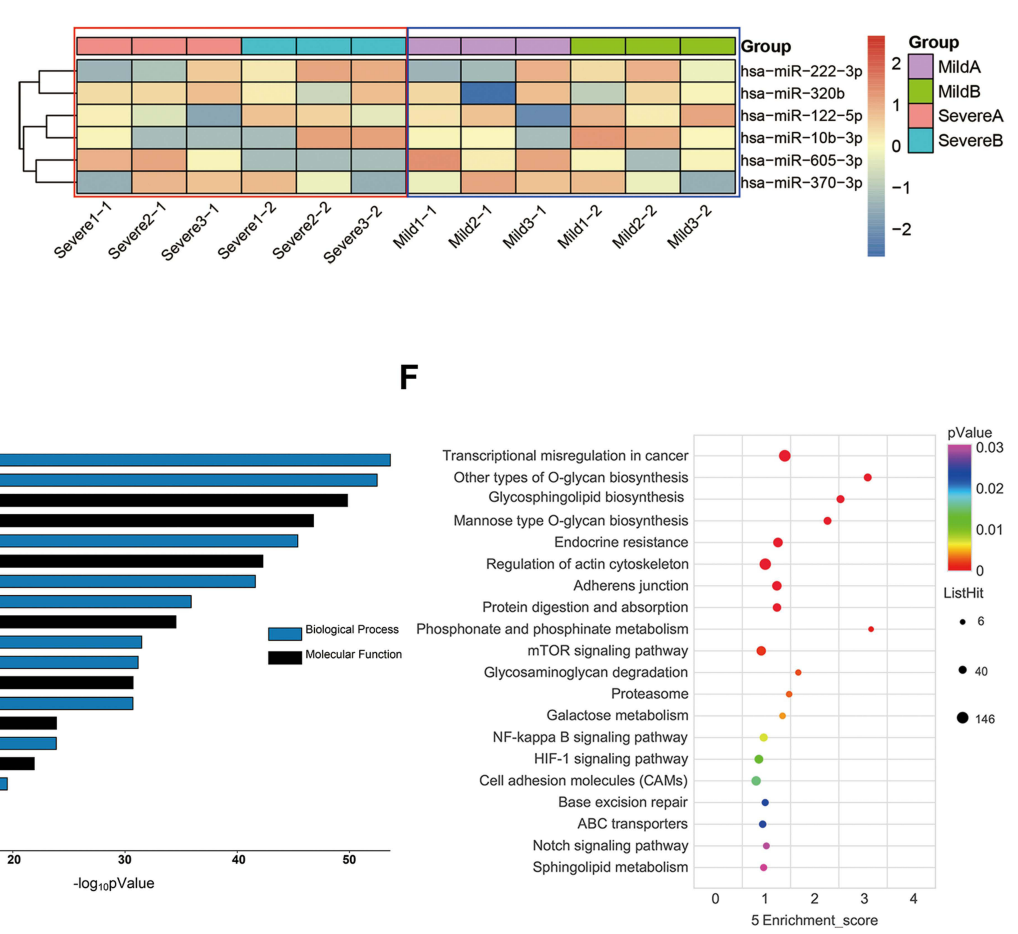

Figure 2 Screening and functional analysis of chemotherapy-related exosomal miRNAs. (A and B) Differential expression of exosomal miRNAs in different groups of patients before and after chemotherapy. (C and D) Venn diagram and heatmap analysis of differentially expressed exosomal miRNAs in different groups of patients with a common expression trend before and after chemotherapy. (E and F) GO and KEGG analyses of the functions of differentially expressed exosomal miRNAs.

\section{Target Gene Prediction and Functional Analysis of Exosomal miR-122-5p}

We further analyzed the functions of target genes of miR-122-5p through GO and KEGG analyses and determined that miR-122-5p is related to the cellular response to hypoxia, mRNA binding, regulation of the protein response and other functions, especially those related to the cell cycle (Figure 6A and B). miR-122$5 p$ has multiple target genes, such as SMAD2, RBL1, SMC1A, and CTDP1; in particular, the target gene 
A

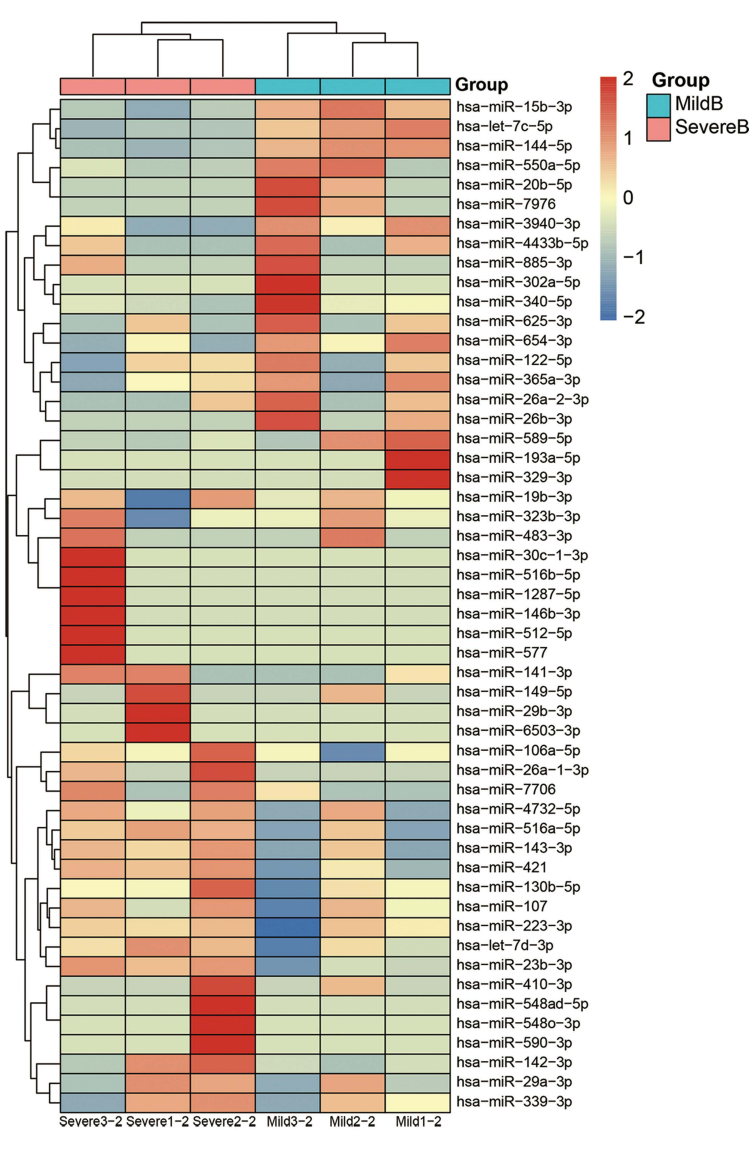

B

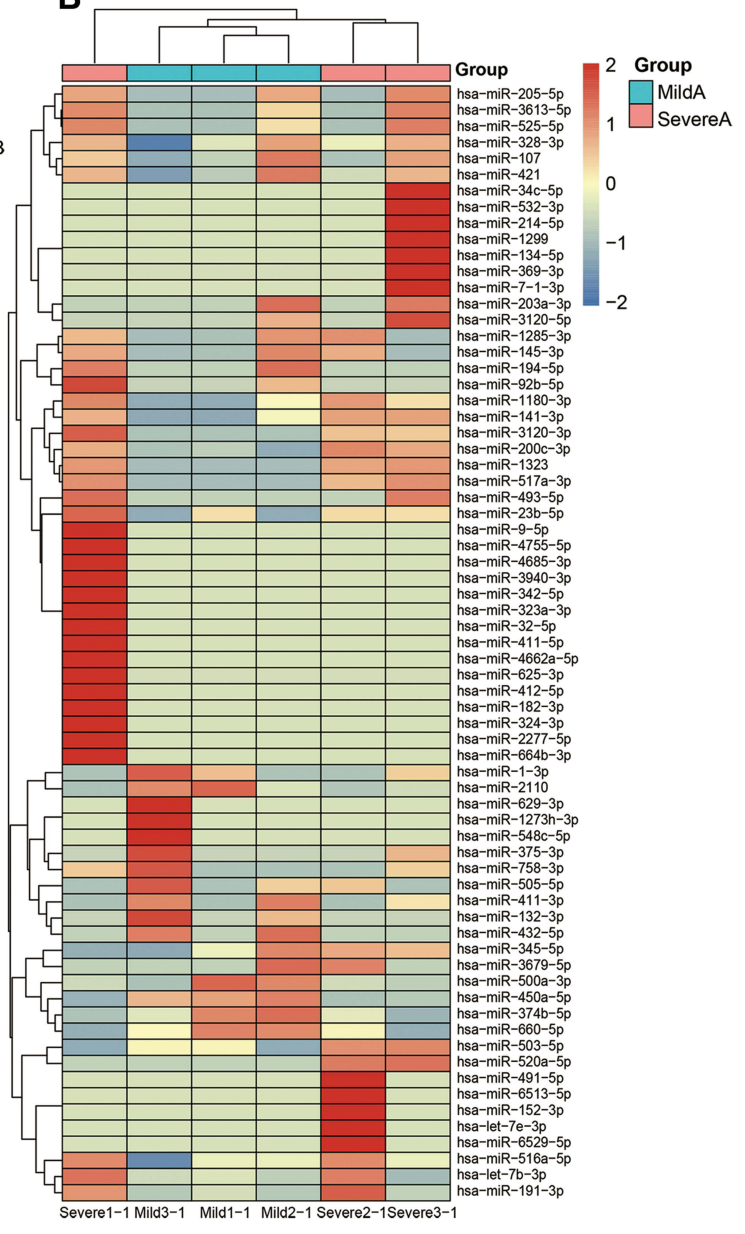

C

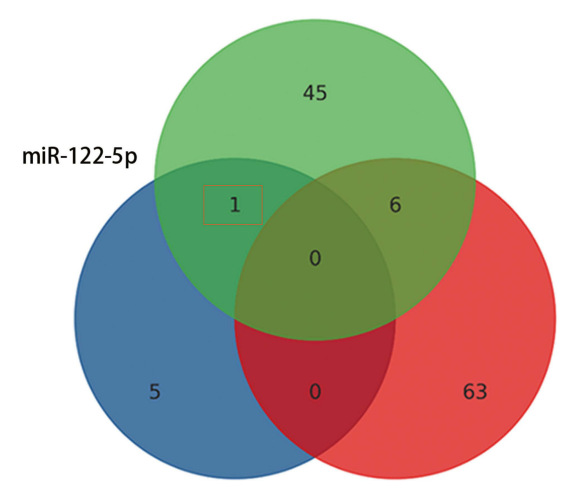

Figure 3 Screening and functional analysis of myelosuppression-related exosomal miR-122-5p. (A) Small RNA sequencing was performed to detect levels of exosomal miRNAs in colorectal cancer patients with mild and severe myelosuppression after chemotherapy. (B) Small RNA sequencing was performed to detect levels of exosomal miRNAs in colorectal cancer patients with mild and severe myelosuppression before chemotherapy. (C) A Venn diagram was built to screen differentially expressed exosomal miRNAs.

CDK4 is known to regulate cell cycle progression (Figure 6C). Moreover, CDK4 interacts with other target genes. We explored miR-122-5p and CDK4 sequence- binding sites in the UTR of CDK4 (Figure 6D) and found that miR-122-5p may target this gene to inhibit cell cycle progression. 


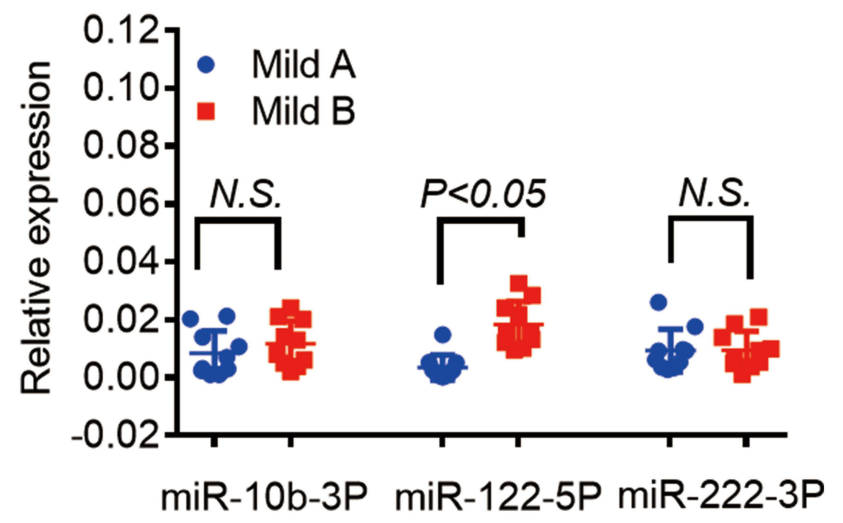

B

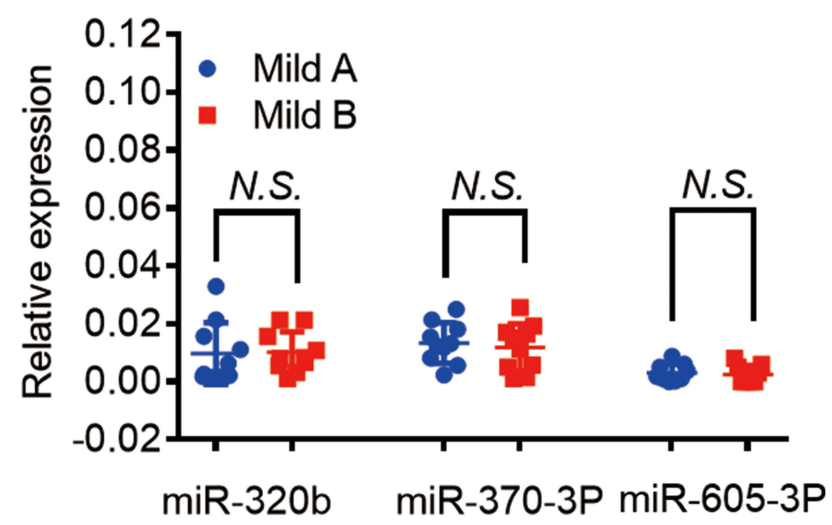

C

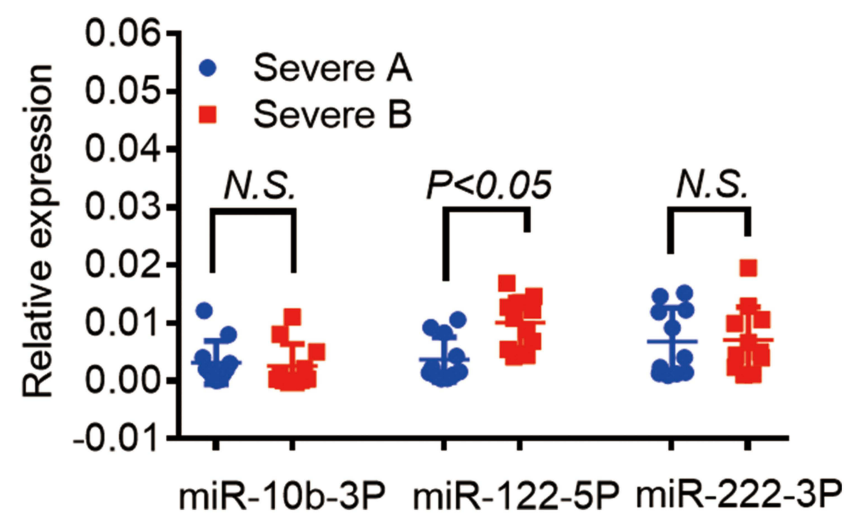

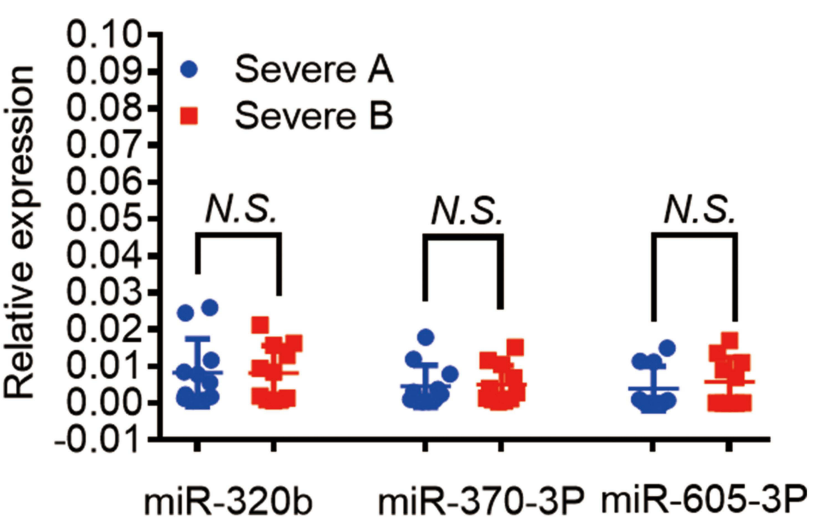

Figure 4 Validation of chemotherapy-related exosomal miRNAs by RT-qPCR. (A and B) Detection of differentially expressed exosomal miRNAs in colorectal cancer patients with mild myelosuppression before and after chemotherapy by RT-qPCR. (C and D) Detection of differentially expressed exosomal miRNAs in colorectal cancer patients with severe myelosuppression before and after chemotherapy by RT-qPCR.

\section{Discussion}

Anticancer chemotherapy usually targets rapidly dividing cells, and it is inevitable that rapidly dividing normal cells might be damaged. Therefore, a serious side effect of this therapy is the occurrence of myelosuppression. ${ }^{16,17} \mathrm{We}$ collected serum samples from 6 patients with colorectal cancer and divided them into SevereA, SevereB, MildA, and MildB groups. We analyzed differentially expressed exosomal miRNAs between groups by performing small RNA sequencing and then screened exosomal miRNAs that showed the same changes before and after chemotherapy in groups (SevereA and SevereB and MildA and MildB) through a Venn diagram. The analyses revealed three downregulated (miR-320b, miR-370-3p, and miR605-3p) and three upregulated (miR-10b-3p, miR-122-5p, and miR-222-3p) exosomal miRNAs, which we further investigated.
To understand exosomal miRNAs related to myelosuppression, we examined differentially expressed exosomal RNAs between the MildA and SevereA groups and between the MildB and SevereB groups. In addition, based on previous results, we screened exosomal miRNAs related to myelosuppression after chemotherapy using a Venn diagram. Although expression of exosomal miR-122-5p was not notably different between the MildA and SevereA groups, it did differ between the MildB and SevereB groups. Specifically, the level of exosomal miR$122-5 p$ in the SevereB group was lower than that in the MildB group after chemotherapy, whereas other exosomal miRNAs were not significantly different. RT-qPCR was used to verify differential expression of serum exosomal miRNAs in patients with different degrees of myelosuppression. We confirmed the results of the previous analysis (ie, exosomal miR-122-5p was jointly upregulated after 
A

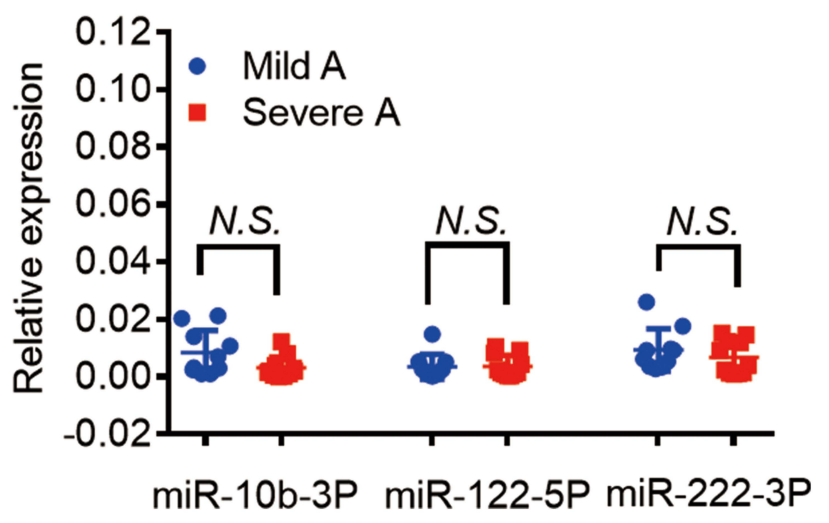

C

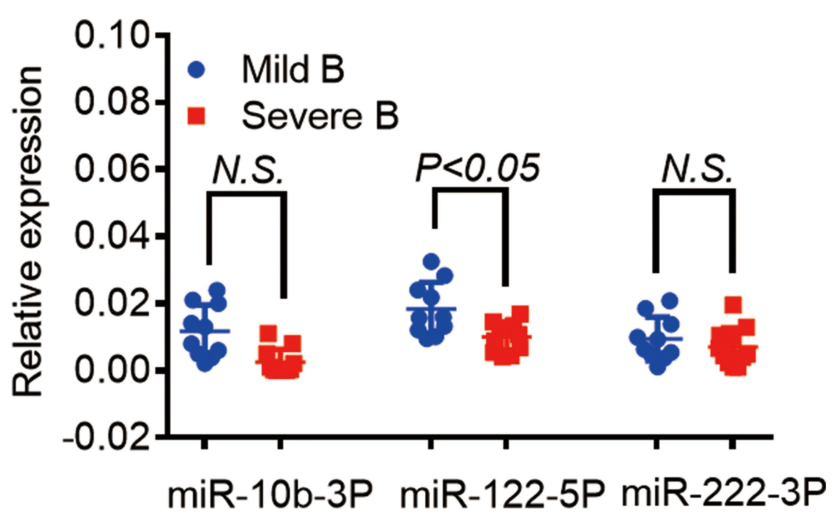

B
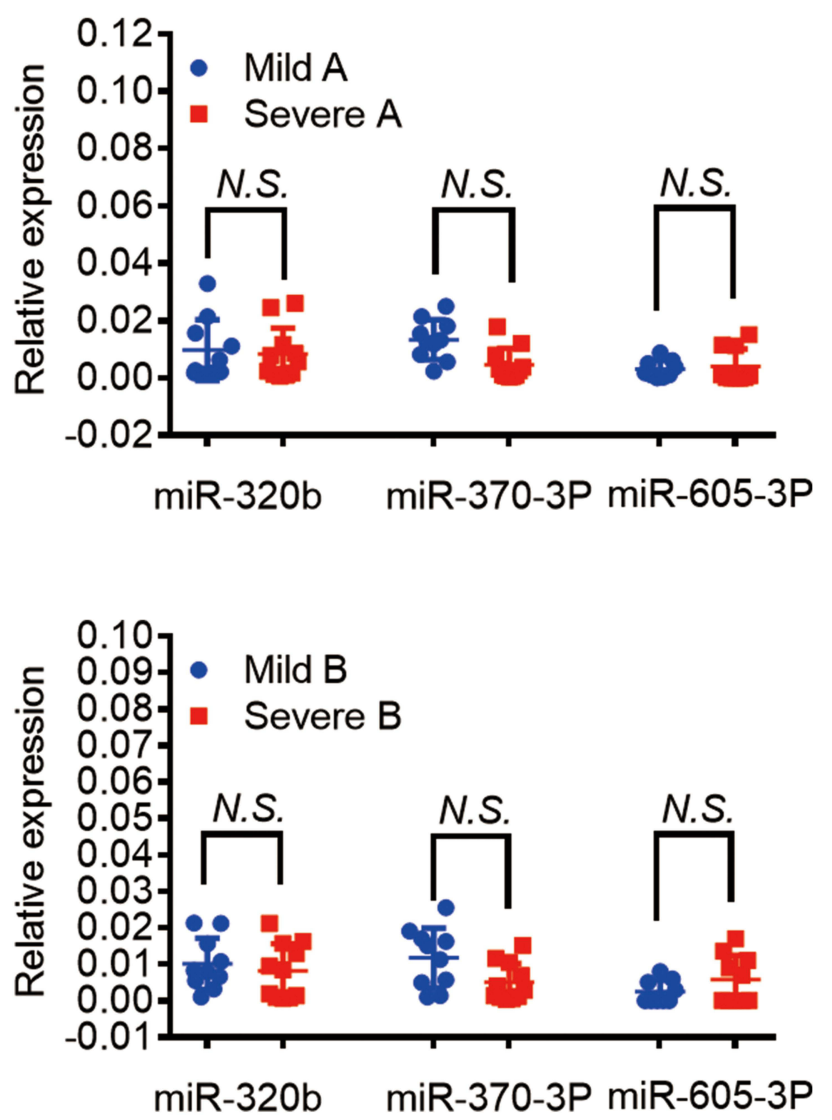

Figure 5 Validation of myelosuppression-related exosomal miR-122-5p by RT-qPCR. (A and B) Comparison of exosomal miRNA expression levels before chemotherapy in colorectal cancer patients with mild and severe myelosuppression by RT-qPCR. (C and D) Comparison of exosomal miRNA expression levels after chemotherapy in colorectal cancer patients with mild and severe myelosuppression by RT-qPCR.

chemotherapy in the two groups); the level of exosomal miR-122-5p was not different between the two groups of patients before chemotherapy, but it was significantly different after chemotherapy. Through verification in patient sera verification, we speculate that patients with high expression have milder myelosuppression after chemotherapy and that patients with low expression have more severe myelosuppression after chemotherapy. The above research results indicate that the level of exosomal miR$122-5 p$ in the serum is closely related to the degree of myelosuppression after chemotherapy.

The cell cycle is a highly conserved and regulated process consisting of four distinct phases. Cyclinindependent kinase (CDK) regulates this process. The checkpoint from $\mathrm{G} 1$ to $\mathrm{S}$ is the key restriction point for cell division. Throughout G1, expression of D-type cyclins (D1, D2, and D3) is enhanced until active complexes with CDK4/6 are formed. These active CDK4/6 complexes partially phosphorylate $\mathrm{RB}$, inhibiting the transcription factor
E2F and leading to separation of it from the Rb-E2F complex and activation of a series of genes, driving entry into $\mathrm{S}$ phase. ${ }^{18}$ The use of certain measures to maintain hematopoietic stem cells/progenitor cells in G0/G1 phase delays the cell cycle and achieves long-term maintenance of the self-renewal and differentiation potential of these cells in vitro. Studies have shown that small molecule CDK4/6 inhibitors temporarily and reversibly block hematopoietic stem cells in G1 phase. In the early stage of chemotherapy (1-2 days after the effect of chemotherapy drugs), although they cannot block the inhibitory effect of chemotherapy drugs on the bone marrow, they can promote the rapid recovery of bone marrow suppression, reduce the depletion of hematopoietic stem cells by chemotherapy, and promote the long-term survival and differentiation of hematopoietic stem cells. ${ }^{19}$ Another study showed that selective inhibition of CDK4/6 can reversibly inhibit bone marrow hematopoietic progenitor cells in G1 phase, reduce DNA damage to hematopoietic progenitor cells (HPCs) caused by 
A

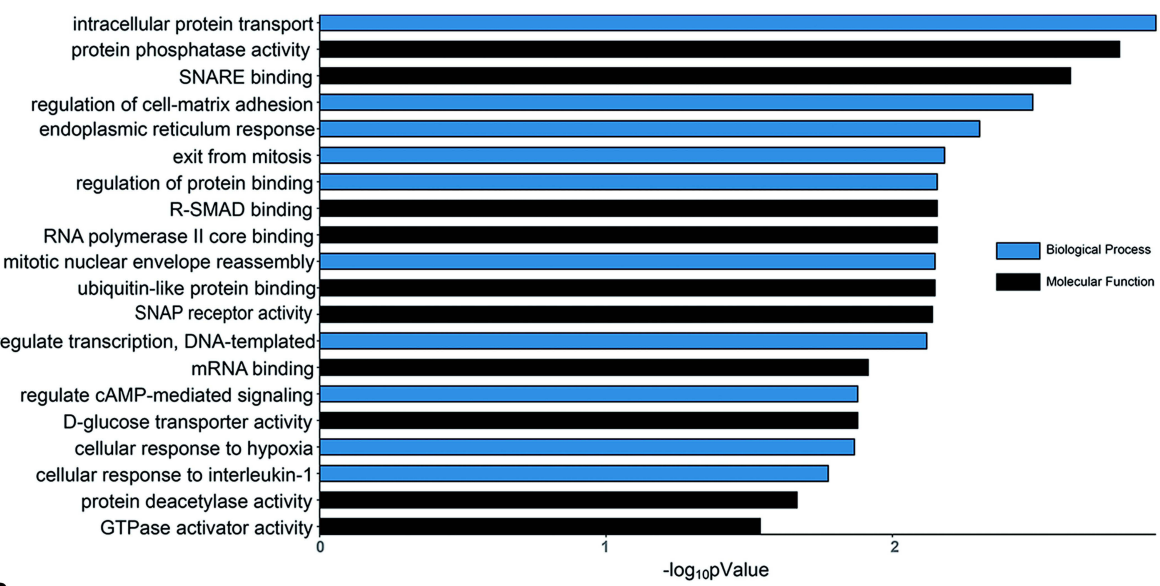

B

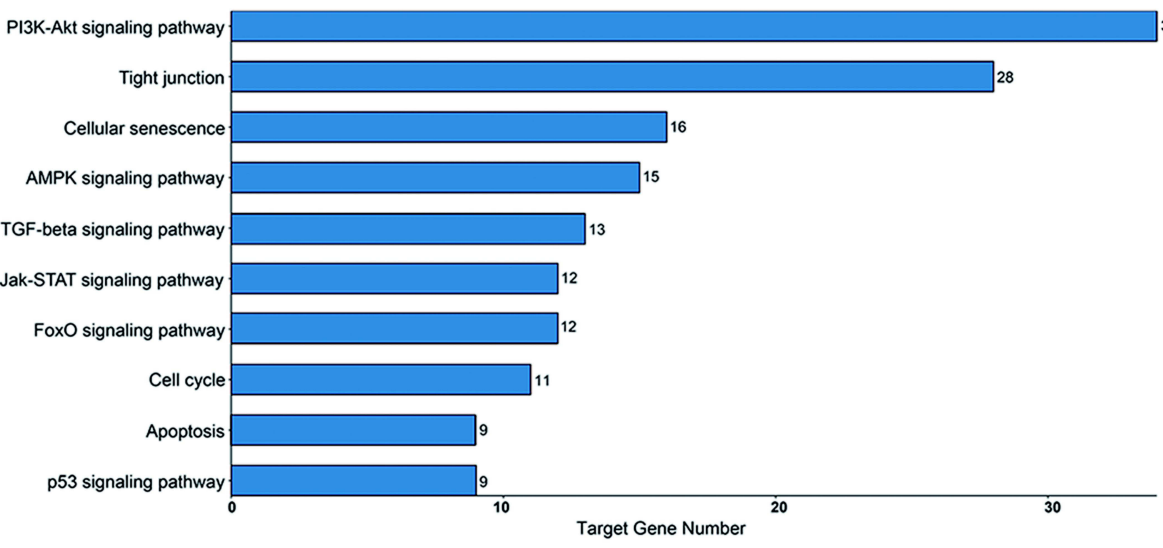

C

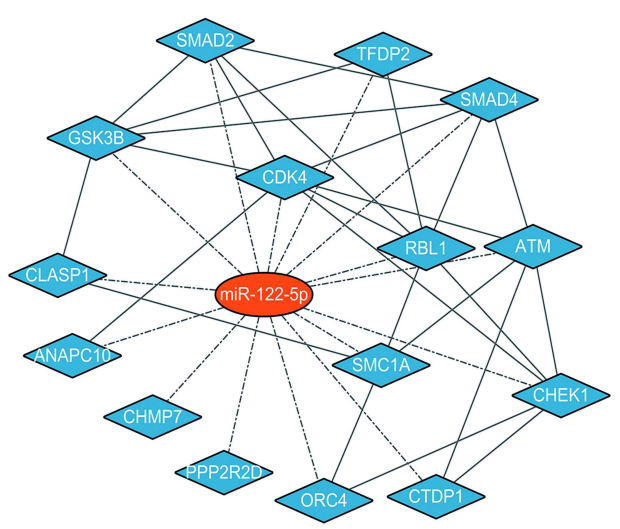

D

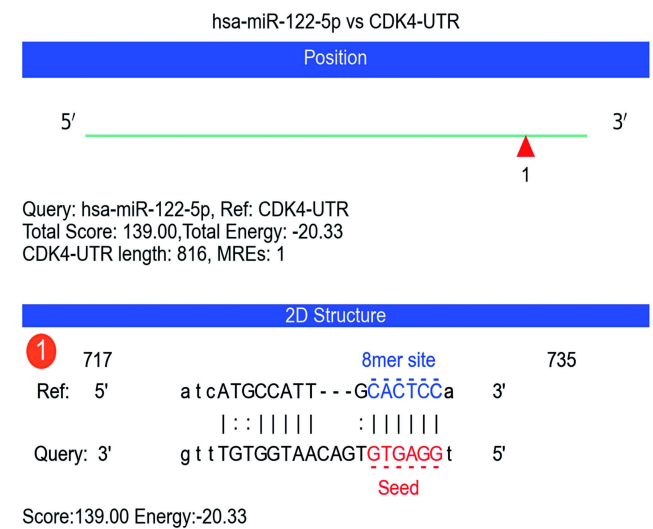

Figure 6 Target gene prediction and functional analysis of exosomal miR-122-5p. (A and B) GO and KEGG analyses of the function of exosomal miR-122-5p. (C) Interaction network diagram of miR-122-5p and its target. (D) miR-I22-5p and CDK4 binding sites.

radiotherapy, and downregulate expression of the DNA damage markers p- $\gamma \mathrm{H} 2 \mathrm{AX}$ and p-P53. ${ }^{20}$ In summary, inhibitors of CDK4/6 specifically block the cell cycle, not only showing good efficacy in a variety of tumor treatments but also becoming a new target for tumor treatment. ${ }^{21}$ Overall, CDK4/6 inhibitors are expected to become targets for reducing myelosuppression after chemotherapy.
Knowledge of epigenetic regulation by miRNAs is advancing. miRNAs are vital to the cell cycle by upregulating or downregulating cell cycle regulators (including cyclin, CDK and $\mathrm{CKI}$ ). We found through GO and KEGG analyses that miR-122-5p has many functions and is closely related to the cell cycle and that CDK4 is a target gene of miR-122-5p. $\mathrm{CDK} 4$ is a cycle-regulating protein that regulates cell cycle 
progression. CDK4 is also closely related to other target genes of miR-122-5p. Notably, studies have shown that miR-122-5p mediates fluoride-induced osteoblast activation by targeting $\mathrm{CDK} 4 .^{22}$ In addition, some reports have shown that miR-122-5p inhibits the proliferation and invasion of bile duct carcinoma cells and promotes apoptosis by targeting expression of ALDOA. ALDOA/miR-122-5p may become a key molecular target for the treatment of bile duct cancer. $^{23}$ In breast cancer, miR-122-5p has been reported to have a tumor-suppressive effect with a positive correlation, ${ }^{24}$ and miR-122-5p acts as a novel regulator of breast cancer cell apoptosis and cell cycle arrest by targeting Bcl-2 and CDKs. ${ }^{25}$ Finally, we explored binding sites of miR-122-5p and its target gene CDK4. Overall, our study shows that exosomal miR-122-5p is associated with the degree of myelosuppression after chemotherapy and that it may target CDK4 to inhibit cell cycle progression.

Nevertheless, some limitations should be addressed. For example, the sample size of our study may not be large enough, which may have limited the statistical power. At present, enhancing efficacy and reducing toxicity are some of the huge challenges of cancer treatment. The current study reveals the potential of exosomal miRNAs related to myelosuppression after chemotherapy in patients with colorectal cancer, and further investigation is needed to assess their utility.

\section{Conclusion}

In summary, the expression level of exosomal miR-122-5p in the serum of patients with colorectal cancer is related to the severity of myelosuppression. Additionally, exosomal miR-122-5p may target CDK4 to inhibit cell cycle progression. The therapeutic targets studied in this work have great potential for the treatment of chemotherapy-induced myelosuppression in patients with colorectal cancer.

\section{Abbreviations}

miRNAs, microRNAs; RBC, Red blood cells; WBC, white blood cells; CSF, colony-stimulating factor; G-CSF, granulocyte colony-stimulating factor; G-M-CSF: granulocytemacrophage colony-stimulating factor; KPS, Karnofsky performance status; snRNAs, small nuclear RNAs; MildA, mild myelosuppression before chemotherapy; MildB, mild myelosuppression after chemotherapy; SevereA, severe myelosuppression before chemotherapy; SevereB, severe myelosuppression after chemotherapy; CDK, cyclinindependent kinase.

\section{Data Sharing Statement}

The data used to support the findings of this study are included within the article.

\section{Ethics Approval and Consent to Participate}

The present study was approved by the Ethics Committee of Hospital of Shanghai University of TCM. The patients who participated in this research signed informed consent, and complete clinical data were available.

\section{Acknowledgments}

The authors thank Fan Zhang (OE Biotech Co., Ltd. (Shanghai, China)) for academic and technical assistance.

\section{Author Contributions}

All authors made a significant contribution to the work reported, including the conception, study design, execution, acquisition of data, analysis and interpretation, or in all of these areas. All authors also participated in drafting, revising, and critically reviewing the article and gave final approval of the version to be published. All authors have agreed on the journal to which the article be submitted and agree to be accountable for all aspects of the work.

\section{Funding}

The present study was supported by the Independent Innovation Project in Putuo District (ptkwws201701), Clinical Specialized Disease Construction Project of Shanghai Putuo District Municipal Health Commission (NO. 2020tszb03), National Nature Science Foundation of China (81873137), Natural Science Foundation of Shanghai (20ZR1450500), Putuo District Health system (ptkwws201905) and Hospital Scientific Research Project (2020366A).

\section{Disclosure}

All authors declare that there are no conflicts of interest in this study.

\section{References}

1. Bray F, Ferlay J, Soerjomataram I, et al. Global cancer statistics 2018: GLOBOCAN estimates of incidence and mortality worldwide for 36 cancers in 185 countries. CA Cancer J Clin. 2018;68(6):394-424. doi:10.3322/caac. 21492

2. Chen W, Zheng R, Baade PD, et al. Cancer statistics in China, 2015. CA Cancer J Clin. 2016;66(2):115-132. doi:10.3322/caac.21338

3. Siegel R, Miller K, Jemal A. Cancer statistics, 2015. CA Cancer J Clin. 2015;65(1):5-29. doi:10.3322/caac.21254 
4. Kelland L, Clarke J, McKeage M. Advances in platinum complex cancer chemotherapy. Platinum Metals Rev. 1992;36:178-184.

5. Akaza H, Saijo N, Aiba K, et al. Platinum compounds in cancer therapypast, present, and future. Gan ToKagaku Ryoho. 2001;28(5):625-635.

6. Simons M, Raposo G. Exosomes-vesicular carriers for intercellular communication. CurrOpin Cell Biol. 2009;21(4):575-581. doi:10.1016/j.ceb.2009.03.007

7. Mathivanan S, Ji H, Simpson RJ. Exosomes: extracellular organelles important in intercellular communication. J Proteomics. 2010;73 (10):1907-1920. doi:10.1016/j.jprot.2010.06.006

8. Gross JC, Chaudhary V, Bartscherer K, et al. Active Wnt proteins are secreted on exosomes. Nat Cell Biol. 2012;14(10):1036-1045. doi:10.1038/ncb2574

9. Sato-Kuwabara Y, Melo SA, Soares FA, et al. The fusion of two worlds: non-coding RNAs and extracellular vesicles-diagnostic and therapeutic implications (Review). Int J Oncol. 2015;46(1):17-27. doi:10.3892/ijo.2014.2712

10. Ogata-Kawata H, Izumiya M, Kuriokaet D, et al. Circulating exosomal microRNAs as biomarkers of colon cancer. PLoS One. 2014;9 (4):e92921. doi:10.1371/journal.pone.0092921

11. Huang XY, Yuan TZ, Tschannen M, et al. Characterization of human plasma-derived exosomal RNAs by deep sequencing. BMC Genomics. 2013;14:319. doi:10.1186/14712164-14-319

12. Song SJ, Ito $\mathrm{K}$, Ala $\mathrm{U}$, et al. The oncogenic microRNA miR-22 targets the TET2 tumor suppressor to Promote hematopoietic stem cell self-renewal and transformation. Cell Stem Cell. 2013;13 (1):87-101. doi:10.1016/j.stem.2013.06.003

13. Garofalo M, Croce CM. Role of microRNAs in maintaining cancer stem cells. Adv DrugDeliv Rev. 2015;81:53-61. doi:10.1016/j. addr.2014.11.014

14. Tanno T, Zhang P, Lazarski CA, et al. An aptamer-based targeted delivery of miR-26a protects mice against chemotherapy toxicity while suppressing tumor growth. Blood Adv. 2017;1(15):1107-1119. doi:10.1182/bloodadvances.2017004705

15. Kloten V, Neumann MHD, Di Pasquale F, et al. Multicenter Evaluation of Circulating Plasma MicroRNA Extraction Technologies for the Development of Clinically Feasible Reverse Transcription Quantitative PCR and Next-Generation Sequencing Analytical Work Flows. Clin Chem. 2019;65(9):1132-1140. doi:10.1373/clinchem.2019.303271.
16. Hassan MN, Waller EK. Treating chemotherapy-induced thrombocytopenia: is it time for oncologists to use thrombopoietin agonists? Oncology. 2015;29(4):295-296.

17. Barreto JN, McCullough KB, Ice LL, et al. Antineoplastic agents and then associated myelosuppressive effects: a review. J Pharm Pract. 2014;27(5):440-446. doi:10.1177/0897190014546108

18. Hao S, Chen C, Cheng T. Cell cycle regulation of hematopoietic stem or progenitor cells. Int $J$ Hematol. 2016;103(5):487-497. doi:10.1007/s12185-016-1984-4

19. He SH, Roberts PJ, Sorrentino JA, et al. Transient CDK4/6 inhibition protects hematopoietic stem cells from chemotherapy-induced exhaustion. Sci Transl Med. 2017;9(387):eaal3986. doi:10.1126/scitranslmed.aal3986

20. Johnson SM, Torrice CD, Bell JF, et al. Mitigation of hematologic radiation toxicity in mice through pharmacological quiescence induced by CDK4/6 inhibition. $J$ Clin Invest. 2010;120 (7):2528-2536. doi:10.1172/JCI41402

21. Chou J, Quigley DA, Robinsonet TM, et al. Transcription-associated cyclin-dependent kinases as targets and biomarkers for cancer therapy. Cancer Discov. 2020;10(3):351-370. doi:10.1158/21598290.CD-19-0528

22. Li C, Qin Y, Yang TO, et al. miR-122-5p Mediates Fluoride-Induced Osteoblast Activation by Targeting CDK4. Biol Trace Elem Res. 2021;199(4):1215-1227. doi:10.1007/s12011-020-02239-z

23. $\mathrm{Xu} \mathrm{Z}$, Liu GC, Zhang M, et al. miR-122-5p Inhibits the Proliferation, Invasion and Growth of Bile Duct Carcinoma Cells by Targeting ALDOA. Cell Physiol Biochem. 2018;48(6):2596-2606. doi:10.1159/000492702

24. Venkatadri R, Muni T, Iyer V, et al. Role of apoptosis-related miRNAs in resveratrol induced breast cancer cell death. Cell Death Dis. 2016;7(2):e2104. doi:10.1038/cddis.2016.6

25. Ergun S, Ulasli M, Igci YZ, et al. The association of the expression of miR-122-5p and its target ADAM10 with human breast cancer. $\mathrm{Mol}$ Biol Rep. 2015;42:497-505. doi:10.1007/s11033-014-3793-2

\section{Publish your work in this journal}

Cancer Management and Research is an international, peer-reviewed open access journal focusing on cancer research and the optimal use of preventative and integrated treatment interventions to achieve improved outcomes, enhanced survival and quality of life for the cancer patient.
The manuscript management system is completely online and includes a very quick and fair peer-review system, which is all easy to use. Visit http://www.dovepress.com/testimonials.php to read real quotes from published authors. 